\title{
STUDY OF BIODIVERSITY OF FUNGAL BIOAEROSOLS IN MUMBAI
}

\section{METROPOLIS}

\section{Umesh B. Kakde}

Government Of Maharashtra's Ismail Yusuf College, Jogeshwari (E), Mumbai400060 India

Corresponding author Email : drumeshkakde@gmail.com

\begin{abstract}
:
Fungal spores are the group of particles which are very numerous in the air and, on account of their dimensions (several micrometers), are classed as a bioaerosol. Bioaerosols consists of aerosols containing microorganisms like bacteria, fungi, viruses or organic compounds derived from microorganisms viz., endotoxins, metabolites, toxins and other microbial fragments. Fungal spores form an important constituent of bioaerosol and they are often well adapted to airborne dispersal. Air serves as a mode of transport for the dispersal of bioaerosls from one location to another. In the course of evolution, the fungi have probably exploited the wind for their dispersal more thoroughly than any other group of organisms and consequently dominate the airspora (80\% - 90\%). Many fungal bioaerosols are frequently occur in the air environment, produce mycotoxins noxious for living organisms, and often cause allergic, immunotoxic diseases. Besides these many spores are allergic and have capability to produce the mycotoxins. Most of the fungal spores follow the seasonal dynamics. The outdoor fungal bioaerosols was studied in Mumbai city throughout the year at different locations. The climate of the Mumbai is hot and humid. The maximum spores were of Deuteromycotina (88\%) which is followed by Basidiomycotina (4\%), Ascomycotina (2\%), unidentified spores \& hyphal fragments $(6 \%)$. The most prevalent outdoor airborne fungal spores recorded were Aspergillus/Penicillium type which contributes $\geq 44 \%$ of the total fungal bioaerosols, followed by Cladosporium, Alternaria, Curvualria, Smuts, Uredospores, unidentified spores and hyphal fragments. Most common outdoor genera showed distinct peaks from July to September and showed well defined seasonal patterns while April - June showed the lean period for fungal spores.
\end{abstract}

\section{Keywords:}

Fungal spores; bioaerosols; airborne; seasonal patterns; outdoor; mycotoxins; immunotoxic; allergic 


\section{Introduction:}

Bioaerosols are defined as airborne particles of biological origin. Bioaerosols include airborne bacteria, viruses, fungi, and other biological fragments such as airborne DNA fragments, tissues scales etc. There are two categories of bioaerosols, viable bioaerososls and non-viable bioaerosols. Viable aerosols are metabolically alive and with the potential to reproduce. Only viable aerosols can be infectious or pathogenic. While non-viable biaerosols originates from living organisms but are not currently live thus cannot multiply and can cause allergic or toxic reactions. Fungal spores form an important constituent of bioaerosol and they are often well adapted to airborne dispersal. In the course of evolution, the fungi have probably exploited the wind for their dispersal more thoroughly than any other group of organisms and consequently dominate the airspora (80\%-90\%). About 250,000 species of fungi are known at the present time, of which about 100 have been associated with an allergic pathology as documented by a hypersensitivity skin prick test and/or bronchial or nasal provocative tests. However, the prevalence of sensitization to fungi is not well known, and it has been suggested to vary from 3 to 91\% (Horner et al., 1995). The composition and concentration of airborne fungal spora are largely determined by geographical location, meteorological factors, vegetation, and human activities (Lacey 1981), as well as by a wide range of interrelated environmental and biological factors. The number and types of microorganisms in the air keep changing with the time of day, weather, season, geographical location and the proximity of local sources (Ebner et al., 1989; Inceoglu et al., 1994; Li \& Kendrick, 1995; Kakde et al., 2001). Concentrations of fungal spores in the atmosphere at any particular moment are influenced by the processes involved in their production, release, and deposition (Lyon et al. 1984). Airborne biological particles can affect every aspect of our lives. In the recent years more attention has been given to the allergic fungal spores suspended in various environs and causing health hazards to humans. 
Exposure to bio-aerosols, containing airborne microorganisms and their byproducts, can result in respiratory disorders and other adverse health effects such as infections, hypersensitivity pneumonitis and toxic reactions (Gorny et al., 2002). Fungi are common in indoor and outdoor environments and nearly $10 \%$ of people worldwide have fungal allergy (Pasanen et al., 1996). Fungal infection can also increase the chances of contamination by mycotoxins, which can cause neurological disorders, liver cancers, lung cancers and other diseases. Many of the molds capable of producing mycotoxins are also frequent contaminants of the food and agricultural commodities. Molds, which are of importance in food because of potential mycotoxin production, include members of the genera Aspergillus, Trichothecium, Fusarium. The mycotoxins that are currently receiving the most attention as potential hazards to human and animal health include aflatoxin, ochratoxin A, sterigmatocystin, patulin, penicillic acid, citrinin, zearalenone and the toxic trichothecenes. All these compounds cause some degree of acute toxicity when given in high amounts (Kramer et al. 1960, Hudson 1969, Willie and Morehouse 1978, Bullerman 1979, Wicklow and Shottwell 1983, Miller 1990 \& 1992). More attention is now being paid to the fungal spores in the air and to the human health problems they cause). Little is known about the allergenic potential of the different types of spores and about the levels of exposure capable of provoking allergic responses when they are encountered in outdoor environments (Levetin et al. 1995). No systematic studies on airborne fungal bioaerosols with relation to seasonal variation in the urban and rural areas of Mumbai have been published. However, such information is needed to evaluate the relationship between prevalence of fungal bioaerososl, allergenic disorders to understand the type of mold exposure that can cause health effects.

\section{Material and Method:}

The bioaerosol sampling will be carried out in the rural and urban area to determine the air-borne fungi in Mumbai city. Five different outdoor locations 
were selected for the sampling. The sampling was carried out by both the method i.e. by culture plate exposure \& rotorod sampling. The settle plate method used for the qualitative analysis and for the quantitative analysis was carried out with the help of rotorod air sampler to analyze the spores in per cubic liter of air. The conidia of Aspergillus spp., Penicilium spp. and many other fungi whose conidia or spores are round in shape with similar size would be very difficult to identify using the volumetric method owing to their great morphological similarity. Hence two different culture media were used for the culture plate enumeration to get the proper identification and representation of the culturable fungi. Sampling locations: Five sampling locations were selected in the Mumbai urban area i.e. in East, West, North, and South directions and one sampling location was selected in the centre i.e. in the heart of the city (Fig. 1). Sampling \& Isolations: The rotorod air sampler with a sampling rate of 100 L min-1 (Tilak Air Sampler, 1982) was used to analyze the total spore counts. The sampler was operated for $15 \mathrm{~min}$. at the height of one meter above the ground level at fifteen day intervals. The sampling was done during peak hours i.e. 10 a.m. to 1 p.m. Exposed cellophane tapes were mounted with glycerin jelly and examined under microscope. The air samples were microscopically analyzed and the obtained result expressed as a monthly average of fungal spores $/ \mathrm{m} 3$ of air. The trapped spores were identified with the help of available standard literature (Ainsworth et al., 1973; Tilak, 1989; Nair et al., 1986). To monitor the culturable fungal species at each location two Petri dishes (9 cm diameter) containing Potato Dextrose Agar (PDA) \& $\mathrm{CZ}$ media were exposed in the atmosphere. Two Petri dishes of each medium were exposed for 3 minutes at intervals of 15 days to the level of 1.5 meter from the ground. Temperature, rainfall and humidity changes were also recorded during the sampling. After the completion of the exposure time, the exposed Petri plates were placed in pre-sterile polythene bags and brought back into the laboratory for further analysis. Petri plates were incubated at room temperature $\left(25^{\circ} \mathrm{C}\right)$ for seven days. Fungal colonies were counted and correlated to fungal spore 
contribution. The identification of colonies was based on the colour, size, and shape of the colonies and other morphological features aided by published literatures (Gilman, 1957; Barnett, 1960; Raper and Fenell, 1965; Raper and Thom, 1968; Smith, 1969).

\section{Result and Discussion:}

Twenty - three identifiable spore taxa belonging to various categories were recovered. Of these Ascomycetes with four, Basidiomycetes with three while rest of the spore types were of Deuteromycetes. Besides unidentified spores, the hyphal fragments, algal filaments, insect"s scales, protozoan cysts, pollen grains etc. were also trapped and categorized separately. The list of dominant fungal spore types and its relative concentrations are given in Table 1. The spores of Aspergillus-Penicillium, Cladosporium, Alternaria, Curvularia,

Smuts, Basidiospores, Drechslera, Helminthosporium and Nigrospora trapped with varying frequencies throughout the year. The spores of Aspergillus-

Penicillium group having small, spherical and similar type of spores viz., Aspergillus, Penicillium, Trichoderma, Mucor, Rhizopus etc. were predominant and contributed $\geq 44.5 \%$ to the total air-spora, followed by Cladosporium (16.5\%), Alternaria (8.4\%), Curvularia (5.3\%), Smuts (2.0\%). The other spores like Helminthosporium, Uredospores, Chaetomium, Diplodia, Pithomyces, Torula were also trapped once or many times during the period of investigation. Dominancy of Aspergillus-Penicillum group of spores also confirmed by the results obtained by settle culture Petri plate method. The maximum colonies of Aspergillus species were grown in the culture medium which is followed by the colony forming units (CFU) of the genus Penicillium, Mucor, Rhizopus, Cladosporium, Alternaria, Curvularia, Chaetomium, Trichoderma etc. The most dominant fungal species were Aspergillus niger, A. flavus, A. fumigates, Penicillium chrysogenum and were predominant throughout period of investigation. Other important fungal species viz., Aspergillus ochraceous, A. terreus, , A. versicolor, A. wentii, Alternaria brassicae, A. solani, A. tenuis, 
Botrytis cinerea, Candida albicans, Colletotrichum spp., Curvularia lunata, C. tetramera, Fusarium chlamydosporum, F.moniliforme, F.solani, Helminthosporium oryzae, Nigrospora sphaerica, Penicillium citrinum, P.italicum, Phoma lingam, Trichoderma lignorum, T. viride, Trichothecium roseum, Verticillium glaucum, Cunninghamella spp., Rhizopus stolonifer, Mucor spp., Chaetomium globosum and many sterile fungi were isolated with varying frequencies throughout the year. The other type and fungal hyphe contributed $\geq 6 \%$ of the total aerospora which includes different unidentified spores. Deuteromycetes spores registered an average of $88 \%$ of the total trapped spores and were most common throughout the year followed by Basidiomycetes (4.0\%), Ascomycetes (2\%) (Fig. 2). The fungal spores showed significant seasonal variations. The highest incidence of spores was observed during July-October while January to May was the lean period. The maximum daily incidence of fungal spores were recorded in August (9225 spores/m3) when the temperature and relative humidity ranged between $24.5 \mathrm{oC}$ to $29.3 \mathrm{oC}$ and 86 to 95\% respectively; and the lowest was found in April and May (2125 4185 spore $/ \mathrm{m} 3$ ) when maximum temperature and relative humidity were ranged between 23.7-33.3 oC and 65-71\% respectively (Fig. $3 \& 4$ ). The difference in the spore concentration and types could be due to the prevailing environmental conditions and presence of organic waste matter, plant debris. The release of fungal spores and consequently their concentrations in the atmosphere are the result of the action of many biological and environmental factors. The occurrence of fungal spores in the air is markedly seasonal because of these organisms" sensitivity to weather changes (Hjelmroos 1993, Craig and Levetin 2000, Stepalska \& Wolek 2005). In the last few years it has been reported that the spores and sclerotia of toxic fungi contain very large concentrations of mycotoxins. For determination of their adverse effects on the human health, many studies were carried out about the fungal community both in outdoor and indoor environments. It was reported that the dominant fungal genera were Penicillium, Aspergillus, Alternaria and Cladosporium in 
the atmosphere and their concentrations differed from place to place because of local environmental conditions such as temperature and humidity, fungal substrates and human activities (Shelton et al., 2002). Concentrations of fungal spores in the atmosphere at any particular moment are influenced by the processes involved in their production, release and deposition. The eventual process of spore deposition depends largely on meteorological factors and the size and shape of the spores. The spores of broad range of fungi including Aspergillus-Penicillium type, Cladosporium, Fusarium, Alternaria, Chaetomium, Nigrospora, Torula, Pithomyces were predominant in the air of Mumbai. The most dominant fungal species were Aspergillus niger, A. flavus, A. fumigatus, Pencillium chrysogenum, Trichoderma spp., Chaetomium spp. In the present investigation the concentration of these spores is relatively high as compared to other spore types and also shows the seasonal fluctuations. These findings are in close agreement with other investigations carried out using similar study areas in India as well in other countries (Burge, 1989; Gravesen, 1979; Hudson, 1969; Kakde et al., 2001; Kramer et al.,1960; Lacey, 1981). Exposure to bioaerosols may cause allergies or inflammation of body tissues. Long term effects may result in chronic bronchitis, asthma or alveolitis. Nearly all fungi may be allergenic depending on the exposure situation and dose. The relevant route of exposure is inhalation, and the adverse effects are revealed to duration and intensity of fungal exposure. However typical for allergic reactions is that once an individual develops an allergy to certain fungi, even small airborne concentrations can trigger an asthma attacks or other allergic reactions. Spores of some fungal species (e.g. Alternaria, Cladosporium, Epicoccum, Ganoderma, Aspergillus, Didymella) are known to cause allergic reactions in humans (Burge, 1989; Kendrick, 1990; Lacey, 1991; Kakde et al., 2001). Many other fungi are known to be mycotoxic and it has been stated that inhalation exposure may be just as detrimental ingestion. The adverse effects of inhaled fungal propagules on the immune system have been well documented by various workers (Salvaggio, 1986; Bullerman 1979; Burge, 1989). 


\section{Conclusion:}

Bioaerosols affects people, plants and the environment. Natural and manmade bioerosols present in indoor as well as outdoor environment. The study of seasonal and diurnal rhythm in the occurrence of airborne spores is a basis for further analyses and generalizations. It also is of a great practical importance. This knowledge can be a useful tool in agrobiology, particularly with respect to pathogenic fungi. The control of airborne spores permits a better understanding of the mechanisms in the dispersal and deposition of spores, epidemiology of fungal plant diseases, and the application of suitable plant protection measures. The analysis of data indicates that concentration of airborne fungi in Mumbai city environment is very high and quite variable depending on the climatic conditions. The spores of Aspergillus, Penicillium, Cladosporium were found to be predominant. Seasonal variation of the various types was highly related to the availability of the substrates needed for the fungal growth. The seasonal climate had positive variations of the influence on occurrence of aeromycoflora. Monsoon and winter months registered maximum frequency and concentration of fungal spores due to favorable growth and sporulating conditions for fungi and availability of suitable substrates. Further research is needed in bioaerosols management and its control in different environments.

\section{Acknowledgement:}

The author is highly grateful to University Grant Commission, New Delhi for the financial assistance for major research project.

\section{Reference:}

Ainsworth G.C., Sparrow F.K. and Sussman, A.S. (1973). The Fungi (Vol. IV A). Academic Press, New York and London.

Barnett, H.L. (1960). Illustrated Genera of Imperfect Fungi. 2nd Ed. Burgessn Publishing Co. Minneapolis, 225 p. 
Bullerman, L.B. (1979). Significance of Mycotoxins to Food Safety and Human Health. J. Food Proc. 42(1), 65-86.

Burge, H.A. (1989). Airborne allergenic fungi: classification, nomenclature, and distribution. Immunol. Allergy clin. North Am. 9, 307-317.

Craig, R. \& E. Levetin (2000). Multiyear Study of Ganoderma Aerobiology, Aerobiologia 16 (1) 79-85

Ebner, M. R., Haselwandter, K. \& Frank, A. (1989). Seasonal fluctuations of airborne fungal allergens. Mycological Research 92:170-176.

Gilman, J.C. (1957). A Manual of Soil Fungi. 2nd ed. IOWA State Uni. Press. Ames, $450 \mathrm{p}$.

Gorny, R. \& Dutkiewicz, J. (2002). Bacterial and fungal aerosols in indoor environment in central and eastern European countries. Ann Agric Environ Med, 9:17-13.

Gravesen, S. (1979). Fungi as a census of allergic diseases. J. Allergy 34, 135154.

Hjelmroos, M. (1993). Relationship between airborne fungal spore presence and weather variables. Grana 1993, 32, 40-47.

Horner, W., Helbling, E. A., Salvaggio, J. E. \& Lehrer, S. B. (1995). Fungal Aeroallergens. Clin Microbiol Rev. 8(2): 161-179.

Hudson, H. J. (1969). Aspergilli in the airspora at Cambridge. Trans. Brit. Mycol. Soc. 52, 153-159.

Inceoglu, O., Pinar, N.M., Sakıyan, N. \& Sorkun, K. (1994). Airborne pollen concentration in Ankara, Turkey 1990-1993. Grana, 33: 158-161.

Kakde Umesh, Hemalata Kakde and Aarti Saoji (2001). Seasonal variation of fungal propagules in a fruit market environment, Nagpur (India). International J. Aerobiologia, 17(2): 177-182. 
Kendrick, B. (1990). Fungal allergens. -In Sampling and Identifying Allergenic Pollens and moulds (Ed. E. G. Smith) pp. 41-49. - Blewstone Press, San Antonio (USA).

Kramer, C.L., Pady, S.M., Rogerson, C.T. (1960). Kansas aeromycology -V. Penicillium and Aspergillus. Mycologia 52, 545-551.

Lacey, J. (1991). Aerobiology and health, the role of airborne fungal spores in respiratory disease. In: P.L. Hawksworth (ed), Frontiers in Mycology. C.A.B. International, Kew, U.K.

Lacey, J. (1981). The aerobiology of conidial fungi. - In Biology of Conidial Fungi. v.1. (ed. G. T. Cole \& Kendrick), pp. 373-415. Academic Press, New York.

Levetin, E., Shaughnessy, R., Fisher, E., Ligman, B., Harrison, J. \& Brennan, T. (1995). Indoor Air quality in schools: exposure to fungal allergens. Aerobiologia, 11:27-34.

Li, D.W. \& B. Kendrick (1994). Functional relationships between airborne fungal spores and environmental factors in Kitchener Waterloo, Ontario, as detected by Canonical correspondence analysis. Grana 33: 166-176.

Lyon, E. L., Framer, C. L. \& Eversmeyer, M. G. (1984). Variation of airspora in the atmosphere due to weather conditions. Grana 23: 177-1 81 .

Miller, J.D. (1992). Fungi contaminants in Indoor air. Atmos. Environ. 26A (12), 2173-2178.

Miller, J. D. (1990). Contamination of food by Fusarium toxin studies from Austria-Asia Proceeding. Japanese Association of Mycotoxicology 32, 1724.

Pasanen, A. L., Lappalainen, S., Pasanen, P. (1996). Volatile organic metabolites associated with some toxic fungi and their mycotoxins. Analyst, 121, 1949-1953 
Raper, K.B. and Thom, C. (1968). A Manual of Penicillia. Hafner Publishing Co., New York, 875 p.

Raper, K.B. \& Fennell D.I. (1965). The Genus Aspergillus. The Williams and Wilkins Co., Baltimore, 686 p.

Salvaggio, J.E. (1986). Human symptoms and epidemiology of fungi in the working paper provided to the health and welfare. Canada working group on fungi and indoor air. Environmental Health Directorate. Health and Welfare Canada, Ontario, K1A, OL2.

Shelton, B. G., K. H. Kirkland, W. D. Flanders, \& G. K. Morris (2002). "Profiles of airborne fungi in buildings and outdoor environments in the United States." Applied and Environmental Microbiology 68(4):1743-1753.

Smith, G. (1969). An Introduction to Industrial Mycology. St. Martin"s Press, New York.

Stepalska, D. \& Wolek, J. (2005). Variation of fungal spore concentrations of selected taxa associated to weather conditions in Cracow, Poland in 1997. Aerobiologia 21: 43-52.

Tilak, S.T. (1982). Aerobiology. Vaijayanti Prakashan, „Ushakal" Saraswati colony (West) Aurangabad, $211 \mathrm{p}$.

Tilak, S.T. (1989). Airborne Pollen and Fungal Spores. Vaijayanti Prakashan, Aurangabad, $312 \mathrm{p}$.

Wicklow, D.T. \& Shottwell, O.L. (1983). Intrafungal distribution of aflatoxin among conidia and sclerotia of Aspergillus flavus and A. parasiticus. Can. J. Microbial. 29, 1-5.

Willie, T. D., Morehouse, L. G., 1978: Mycotoxic fungi, mycotoxins, and mycotoxicosis. Mareal Dekkar Inc., New York. 
Table 1: Mean concentration of dominant fungal spore types (frequently isolated) in Mumbai city at different sampling locations

\begin{tabular}{|l|c|c|c|}
\hline \multicolumn{1}{|c|}{ Fungal Spore Taxa } & $\begin{array}{c}\text { Mean } \\
\text { concentration } \\
\text { Range (spore/m3) }\end{array}$ & $\begin{array}{c}\text { Mean } \\
\text { concentration } \\
\text { Range (CFU) }\end{array}$ & Range \\
\hline ASCOMYCETES & & $\mathbf{2 . 0}$ & \\
\hline Chaetomium & 45 & 1.6 & $10-175$ \\
\hline BASIDIOMYCETES & & $\mathbf{4 . 0}$ & \\
\hline Ganoderma & 30 & 1.1 & $0-150$ \\
\hline Smuts & 55 & 2.0 & $10-325$ \\
\hline Uredospores & 25 & 0.9 & $0-250$ \\
\hline DEUTEROMYCETES & & $\mathbf{8 8 . 0}$ & \\
\hline Alternaria & 230 & 8.4 & $45-550$ \\
\hline Aspergillus/ Penicillium* & 1225 & 44.5 & $800-3000$ \\
\hline Bispora & 30 & 1.1 & $0-85$ \\
\hline Cercospora & 45 & 1.6 & $0-115$ \\
\hline Cladosporium & 455 & 16.5 & $115-985$ \\
\hline Curvularia & 145 & 5.3 & $105-600$ \\
\hline Diplodia & 45 & 1.6 & $0-90$ \\
\hline Drechslera & 35 & 1.3 & $05-70$ \\
\hline Helminthosporium & 35 & 1.3 & $0-50$ \\
\hline Nigrospora & 60 & 2.2 & $25-265$ \\
\hline Pithomyces & 55 & 2.0 & $0-185$ \\
\hline Torula & 30 & 1.1 & $5-65$ \\
\hline Hyphal fragments & 40 & 1.5 & $5-95$ \\
\hline Other Type \# & 125 & 4.5 & $65-525$ \\
\hline * & 2750 & 100.0 & \\
\hline *small, spherical and similar type of spores viz., Aspergillus, Penicillium, \\
\#richoderma, Mucor, Rhizopus etc. & & & \\
\# Unidentified spores & \multicolumn{3}{|c|}{} \\
\hline
\end{tabular}



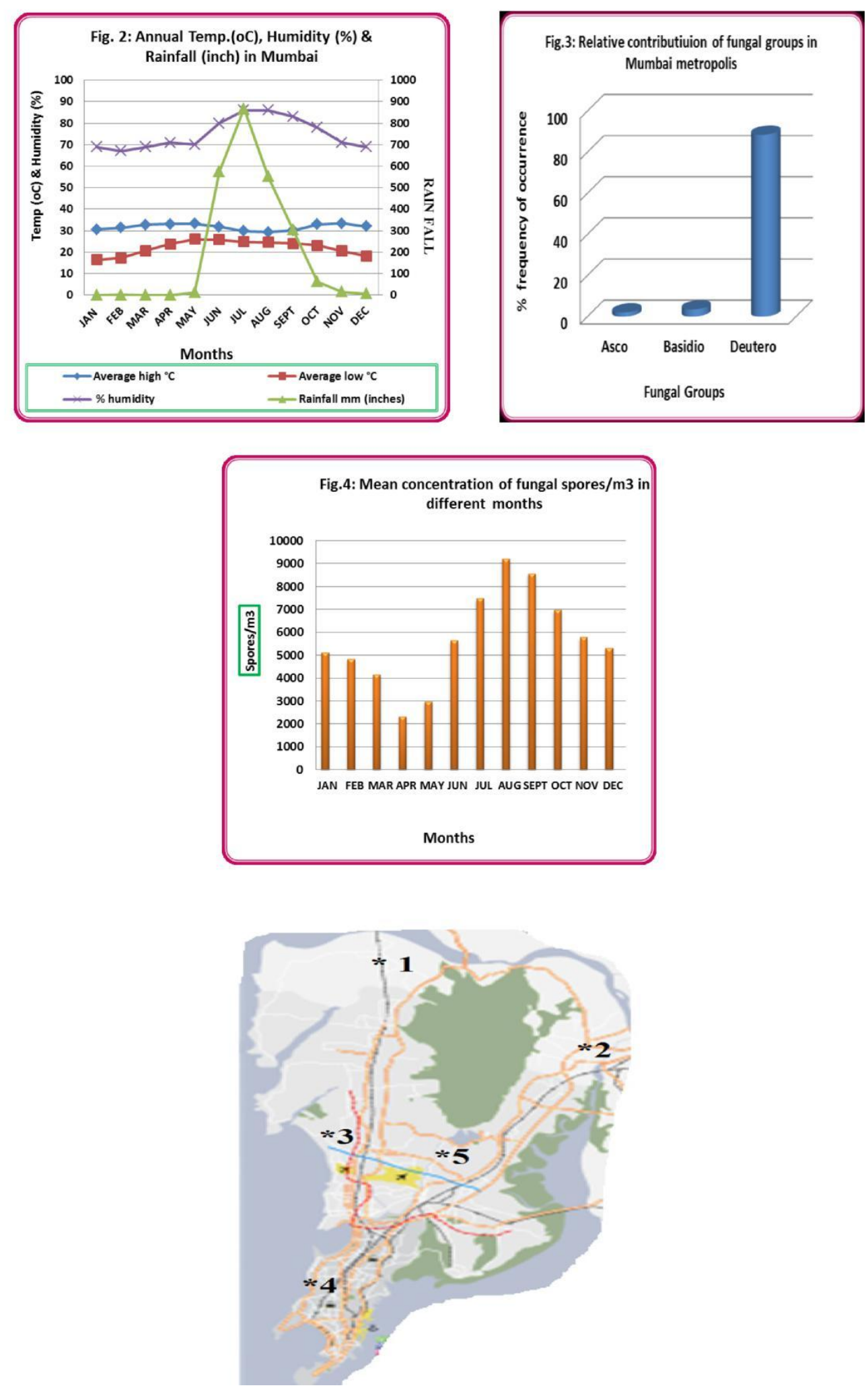

Figure 1 : Sampling Locations in Mumbai Metropolis 